\title{
MiR-485-3p and miR-485-5p suppress breast cancer cell metastasis by inhibiting PGC-1a expression
}

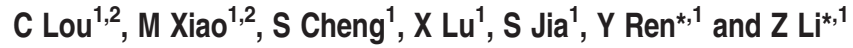

Breast cancer is the worldwide leading cause of cancer mortality in women. The majority of deaths from breast cancer arise from metastasis of local tumors. Cancer cells support their rapid proliferation by diverting metabolites into anabolic pathways, but during cancer metastasis, the proliferative program of invasive cancer cells is suspended for a migratory phenotype. In this study, we demonstrated that both mature forms of miRNA-485, miR-485-3p and miR-485-5p were involved in regulating mitochondrial respiration, cell migration and cell invasion in breast cancer cells by directly targeting and inhibiting the expression of PGC-1 $\alpha$. Specifically, the expression levels of both miR-485-3p and miR-485-5p were decreased in breast cancer tissues. Overexpression of miR-485-3p and miR-485-5p suppressed mitochondrial respiration and potential for cell migration and invasion in vitro, and also inhibited spontaneous metastasis of breast cancer cells in vivo. The suppression of mitochondrial respiration and cell invasion could be partially relieved by restoration of PGC- $1 \alpha$ expression.

Cell Death and Disease (2016) 7, e2159; doi:10.1038/cddis.2016.27; published online 24 March 2016

Breast cancer is one of the most commonly diagnosed malignant tumors, and is the leading cause of cancer mortality in women worldwide. ${ }^{1}$ A vast majority of deaths in breast cancer arise from metastasis, during which tumor cells proliferate, reorganize and migrate into the circulation, and consequently invade other tissues. ${ }^{2}$ Therefore, understanding the pathogenesis of breast cancer metastasis will facilitate the development of novel strategies for effective treatment of the disease.

In cancer cells, although the metabolic demands are primarily satisfied through the process of aerobic glycolysis, they support their rapid proliferation by diverting metabolites into anabolic pathways to accumulate cellular materials required for largescale cell growth., 3 uring cancer metastasis, the proliferative program of invasive cancer cells is suspended, and these cells acquire a migratory phenotype. ${ }^{5}$ The metabolic requirements for this transition process, however, are not well characterized. In a recent study, it was demonstrated that metastatic cancer cells specifically favored mitochondrial respiration and increased ATP production, and that they made use of the transcription co-activator, PGC-1a, to enhance oxidative phosphorylation, mitochondrial biogenesis and oxygen consumption rates. Loss of PGC-1a in cancer cells was shown to abolish their invasive potential and attenuate metastasis without affecting proliferation and tumor growth. ${ }^{6}$

Emerging evidence has suggested that in addition to proteinencoding genes, non-coding RNAs, especially microRNAs, also have important roles in the pathogenesis of cancer metastasis by regulating the expression of cancer-related genes. ${ }^{7}$ MicroRNAs (miRNAs) are small non-coding RNAs approximately 22 nucleotides in length. Although their detailed mechanism(s) of action is not clear, miRNAs are believed to act primarily in a post-transcriptional manner by binding to the $3^{\prime}$-untranslated region (3'-UTR) of target messenger RNA (mRNA), resulting in mRNA degradation or translational inhibition. ${ }^{8}$ Increasing numbers of studies have indicated that miRNAs are involved in regulating numerous cellular processes, including metabolic homeostasis, cell proliferation and cell apoptosis. ${ }^{9}$ Importantly, aberrant expression of miRNA has been reported in many cancer types, including prostate, ${ }^{10}$ colorectal $^{11}$ and glioma. ${ }^{12}$ The roles miRNAs have in cancer onset, development and metastasis are complicated, because it has been noted that miRNAs can either act as oncogenes by repressing target mRNAs of tumor-suppressor genes or as tumor suppressors by negatively regulating oncogene mRNAs. ${ }^{13}$

In our study, we demonstrated for the first time that the miRNAs, miR-485-3p and miR-485-5p, are involved in regulating mitochondrial respiration, cell migration and cell invasion in breast cancer cells by directly targeting and inhibiting the expression of PGC-1a. Specifically, both miR-485-3p and miR-485-5p were downregulated in breast cancer tissues. Overexpression of miR-485-3p and miR-485$5 p$ suppressed both mitochondrial respiration and potential for cell migration and invasion, and this suppression could be partially relieved by restoration of PGC-1 1 a expression.

\section{Results}

The expression of miR-485-3p and miR-485-5p is downregulated in breast cancer. Although it has been suggested that miR-485 may exhibit some tumor-suppressor

\footnotetext{
${ }^{1}$ Department of Breast Surgery, The Affiliated Tumor Hospital, Harbin Medical University, Harbin, China

*Corresponding author: Y Ren or Z Li, Department of Breast Surgery, The Affiliated Tumor Hospital, Harbin Medical University, 150 Haping Road, Nangang District, Heilongjiang Province, Harbin 150081, China. Tel: +86 451 86298666; Fax: +86 451 86298000; E-mail: $436050261 @ q q . c o m$ or dr_Izg@126.com

${ }^{2}$ These authors contributed equally to this work.

Abbreviations: microRNA, miR/miRNA; mRNA, messenger RNA; 3'-UTR, 3'-untranslated region; DMEM, Dulbecco's modified Eagle's medium; FBS, fetal bovine serum; WT, wild type; MT, mutated; LV, lentivirus; H\&E, hematoxylin and eosin; ANOVA, analysis of variance; mtDNA, mitochondrial DNA

Received 09.9.15; revised 14.12.15; accepted 03.1.15; Edited by G Calin
} 

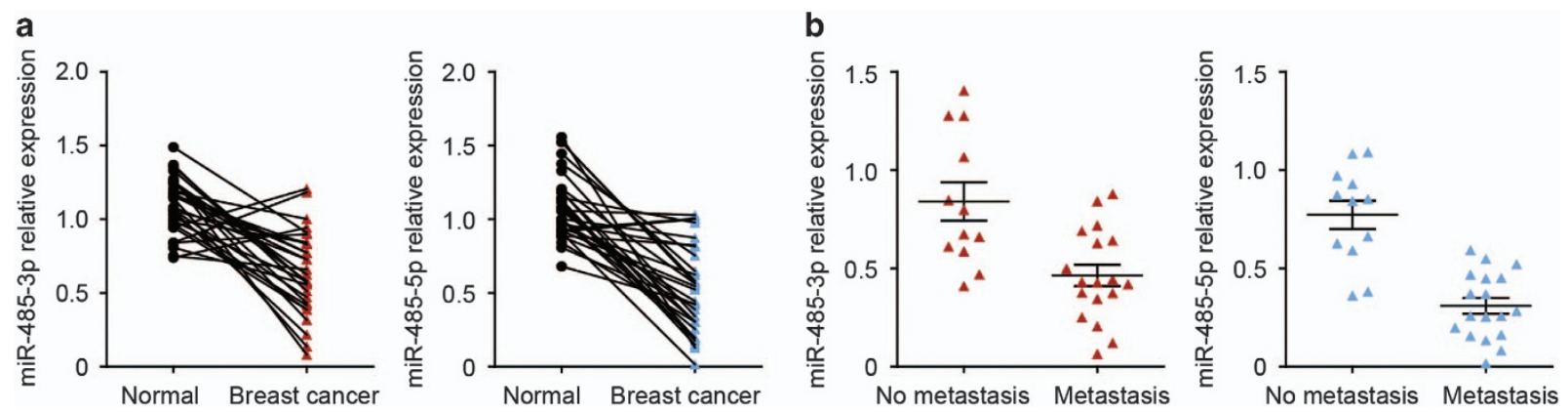

Figure 1 The expression of miR-485-3p and miR-485-5p is downregulated in breast cancer tissues. (a) The expression levels of miR-485-3p and miR-485-5p in cancer tissues compared with adjacent normal tissues in 30 breast cancer patients, $P<0.001$ by paired $t$-test. (b) The expression levels of miR-485-3p and miR-485-5p in breast cancer tissues with and without lymph node metastasis, $P<0.001$ by $t$-test

Table 1 Analysis of the correlation between miR-485-3p, miR-485-5p and clinicopathologic parameters in breast cancer

\begin{tabular}{|c|c|c|c|c|c|c|c|}
\hline \multirow[t]{2}{*}{ Viable } & \multirow[t]{2}{*}{ Cases } & \multicolumn{3}{|c|}{ miR-485-3p } & \multicolumn{3}{|c|}{$\operatorname{miR}-485-5 p$} \\
\hline & & Low & High & $P$-value & Low & High & $P$-value \\
\hline \multicolumn{8}{|l|}{ Age, years } \\
\hline$<50$ & 17 & 7 & 10 & 0.231 & 9 & 8 & 0.500 \\
\hline$\geqslant 50$ & 13 & 8 & 5 & & 6 & 7 & \\
\hline \multicolumn{8}{|l|}{ Tumor size, $\mathrm{cm}$} \\
\hline$<2$ & 9 & 5 & 4 & 0.500 & 5 & 4 & 0.500 \\
\hline$\geqslant 2$ & 21 & 10 & 11 & & 10 & 11 & \\
\hline \multicolumn{8}{|l|}{ Stage } \\
\hline I-II & 14 & 4 & 10 & 0.033 & 3 & 11 & 0.005 \\
\hline III-IV & 16 & 11 & 5 & & 12 & 4 & \\
\hline \multicolumn{8}{|l|}{ Lymph node } \\
\hline No metastasis & 12 & 3 & 9 & 0.030 & 2 & 10 & 0.004 \\
\hline Metastasis & 18 & 12 & 6 & & 13 & 5 & \\
\hline
\end{tabular}

properties in a specific breast carcinoma cell line, ${ }^{14}$ the functional role of miR-485 in breast cancer development remained largely unclear. Therefore, we first sought to determine the levels of miR-485 expression in breast cancer tissues. We found that the expression levels of the mature forms of miR-485, miR-485-3p and miR485-5p were significantly downregulated in breast cancer tissues from 30 patients compared with adjacent normal tissues (Figure 1a). In addition, of the 30 breast cancer cases, there were 18 cases that had spread to lymph nodes. We then compared the expression levels of miR-485-3p and miR-485-5p between the breast cancer cases with and without lymph node metastasis, and determined that the expression levels of miR-485-3p and miR-485-5p were significantly reduced in breast cancer samples with lymph node metastasis (Figure 1b, Table 1). Taken together, these clinical data indicated that the expression levels of miR-485 were negatively correlated with development and metastasis potential of breast cancers, and therefore miR-485 could be involved in the molecular mechanism(s) of breast cancer progression.

PGC-1a is a direct target of miR-485-3p and miR-4855p. In many cases, miRNAs perform biological functions through negative regulation of their target genes. ${ }^{15}$ Using three public prediction algorithms, Targetscan, microRNA.org and DIANA TOOLS, we initially identified six genes (PGC-1a, SENP1, TCF7, Spred2, KLF6 and Cul3) predicted to be targets for miR-485-3p and miR-485-5p (Figure 2a). To validate the prediction, we transfected miR-control, miR-485-3p or miR-485-5p into MCF-7 and MDA-MB-231 cells, and determined the expression levels of the predicted genes by qRT-PCR. We found that miR-485-3p and miR-485$5 p$ transfection led to significantly reduced expression of PGC-1a in both MCF-7 and MDA-MB-231 cells, but there were no significant changes in the expression levels of the other five predicted miR-485 target genes (Figure 2b). Furthermore, western blot analysis also confirmed that transfection of miR-485-3p and miR-485-5p resulted in decreased PGC-1a protein levels in both MCF-7 and MDAMB-231 cells (Figure 2c).

We next identified potential miR-485-3p and miR-485-5p targeting sites in the $3^{\prime}$-UTR of PGC- $1 a$ (Figure $2 d$ ). To confirm the direct targeting sites of miR-485, we constructed reporter plasmids of the PGC-1a 3'-UTR containing either the wild-type or mutant forms of the potential miR-485-3p or miR-485-5p targeting sites (Figure 2e). Using the luciferase reporter assay, we found that miR-485-3p and miR-485-5p reduced the luciferase activity of the reporter plasmids containing their respective wild-type $3^{\prime}-\mathrm{UTR}$ of PGC-1a. In contrast, miR-485-3p and miR-485-5p had no effect on the luciferase activity of the reporter plasmids containing their respective mutated 3'-UTRs of PGC-1a (Figure 2f). To elucidate PGC-1a expression in breast cancer samples and breast cancer tissues with lymph node metastasis, we performed qRT-PCR and found that PGC-1a was downregulated in breast cancer tissues (Supplementary Figure 1). A negative correlation was seen between miR-485 and PGC-1a (Supplementary Figure 2). Collectively, these results showed that miR-485-3p and miR-485-5p directly targeted the $3^{\prime}-$ UTR of PGC-1 $a$ and negatively regulated PGC-1 $a$ expression.

Both miR-485-3p and miR-485-5p regulate mitochondrial respiration, migration, invasion and proliferation of breast cancer cells. It has recently been reported that PGC-1a is involved in regulating mitochondrial function and metastasis of breast cancer cells. ${ }^{6}$ As PGC-1a is a target for miR-485-3p and miR-485-5p, we first sought to investigate the functional roles of $\mathrm{miR}-485-3 p$ and $m i R-485-5 p$ in 
a

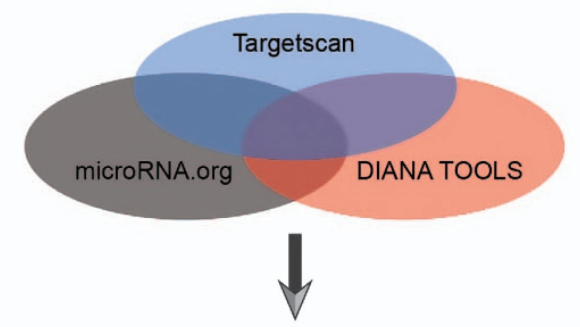

PGC-1 $\alpha$; SENP1; TCF7;

Spred2; KLF6; Cul3

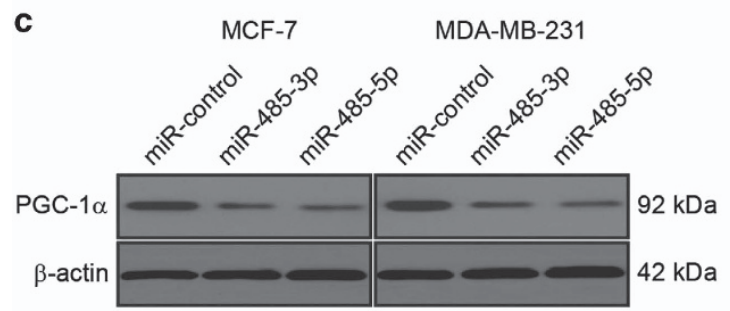

d PGC-1 $\alpha$ 3'UTR WT

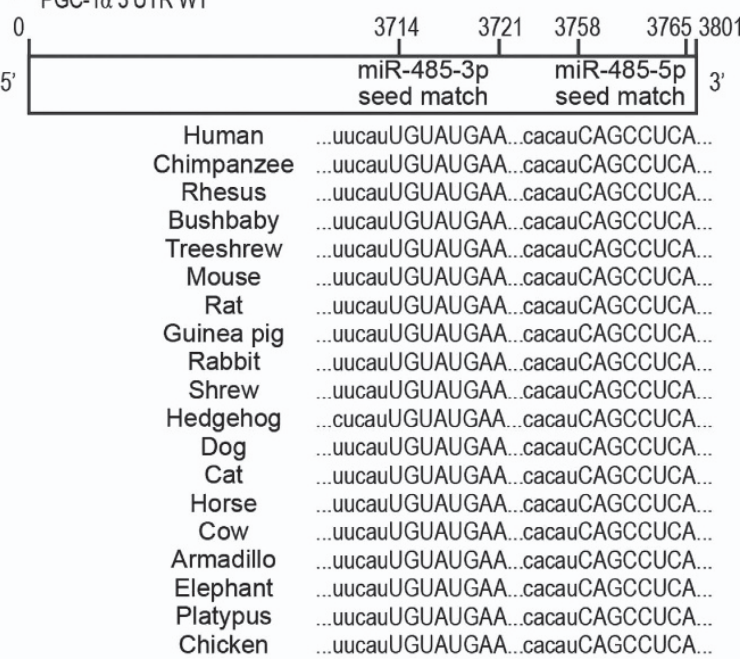

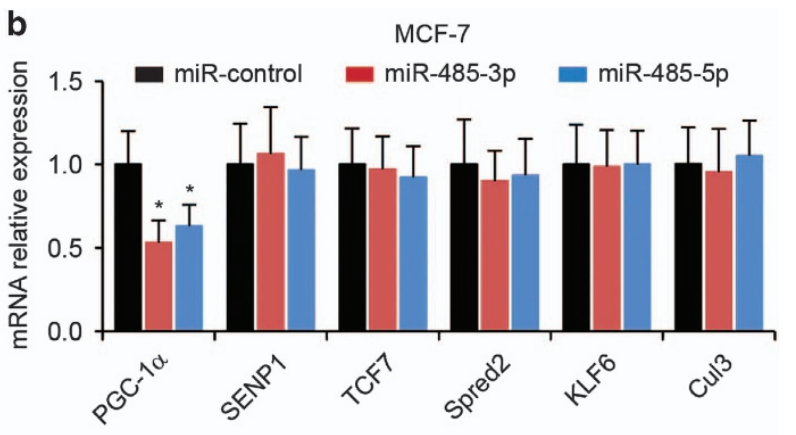

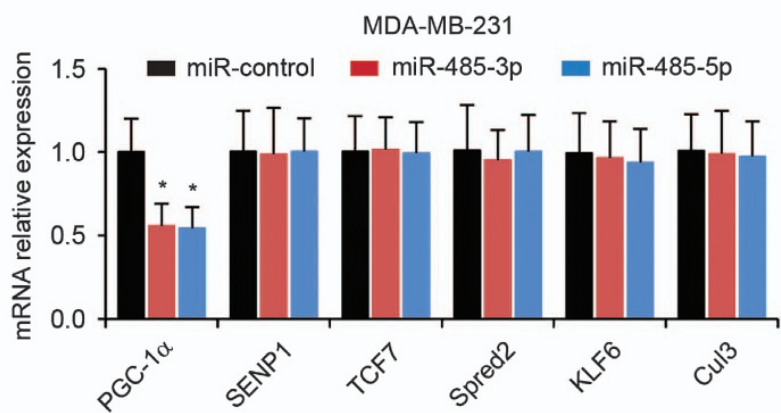

e

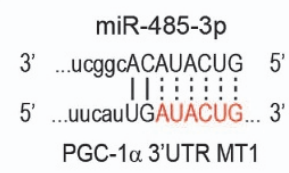

miR-485-5p

3'...ugccgGUCGGAGA 5'

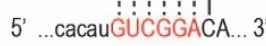

PGC-1 $\alpha$ 3'UTR MT2

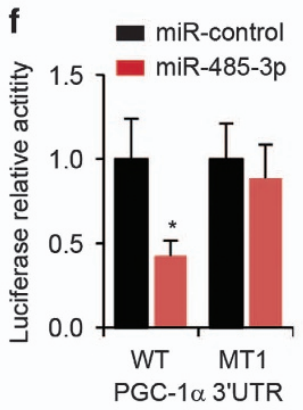

Figure 2 PGC-1 $\alpha$ is a direct target of miR-485-3p and miR-485-5p. (a) Six predicted target genes (PGC-1 $\alpha$, SENP1, TCF7, Spred2, KLF6 and Cul3) of miR-485-3p and miR-485-5p by Targetscan, microRNA.org and DIANA TOOLS. (b) The expression levels of predicted target genes in MCF-7 and MDA-MB-231 cells with transfection of miRcontrol, miR-485-3p or miR-485-5p. $\beta$-Actin was used as an internal control. ${ }^{*} P<0.05$ by one-way ANOVA compared with miR-control-transfected cells. (c) Western blot analysis of PGC-1 $\alpha$ in MCF-7 and MDA-MB-231 cells transfected with miR-control, miR-485-3p or miR-485-5p. $\beta$-Actin was used as loading control. ${ }^{*} P<0.05$ by one-way ANOVA compared with miR-control-transfected cells. (d) Schematic representation of the wild-type PGC-1 $\alpha$ 3'-UTR (WT) showing putative miR-485-3p and miR-485-5p target sites. (e) Mutations of predicted binding sites of miR-485-3p (MT1) or miR-485-5p (MT2) in the PGC-1 $\alpha 3^{\prime}$-UTR. (f) Relative luciferase activity in HEK293T cells co-transfected with WT or MT reporter plasmid and miR-control, miR-485-3p or miR-485-5p. ${ }^{*} P<0.05$ by $t$-test compared with miR-control-transfected cells

regulating mitochondrial respiration of breast cancer cells. We transfected miR-485-3p or miR-485-5p into MCF-7 and MDA-MB-231 breast cancer cells and confirmed overexpression (Figure 3a). We found that overexpression of miR-485$3 p$ and miR-485-5p led to a significant reduction in the expression of two target genes of PGC-1a, ATP5B and ALAS-1, which have important roles in mitochondrial metabolism $^{16}$ (Figure $3 b$ ). Furthermore, we examined the mitochondrial DNA (mtDNA) content and the ATP concentrations in MCF-7 and MDA-MB-231 with miR-485-3p or miR-485-5p overexpression, and found that both miR-485-3p and miR-485-5p significantly reduced the levels of mtDNA content (Figure 3c) and ATP concentrations (Figure 3d).

As PGC-1a was also shown to have a pivotal role in the metastasis of breast cancer cells, ${ }^{6}$ we sought to determine whether miR-485-3p and miR-485-5p are able to regulate migration and invasion of breast cancer cells. We found that overexpression of $\mathrm{miR}-485-3 p$ and $\mathrm{miR}-485-5 p$ significantly 

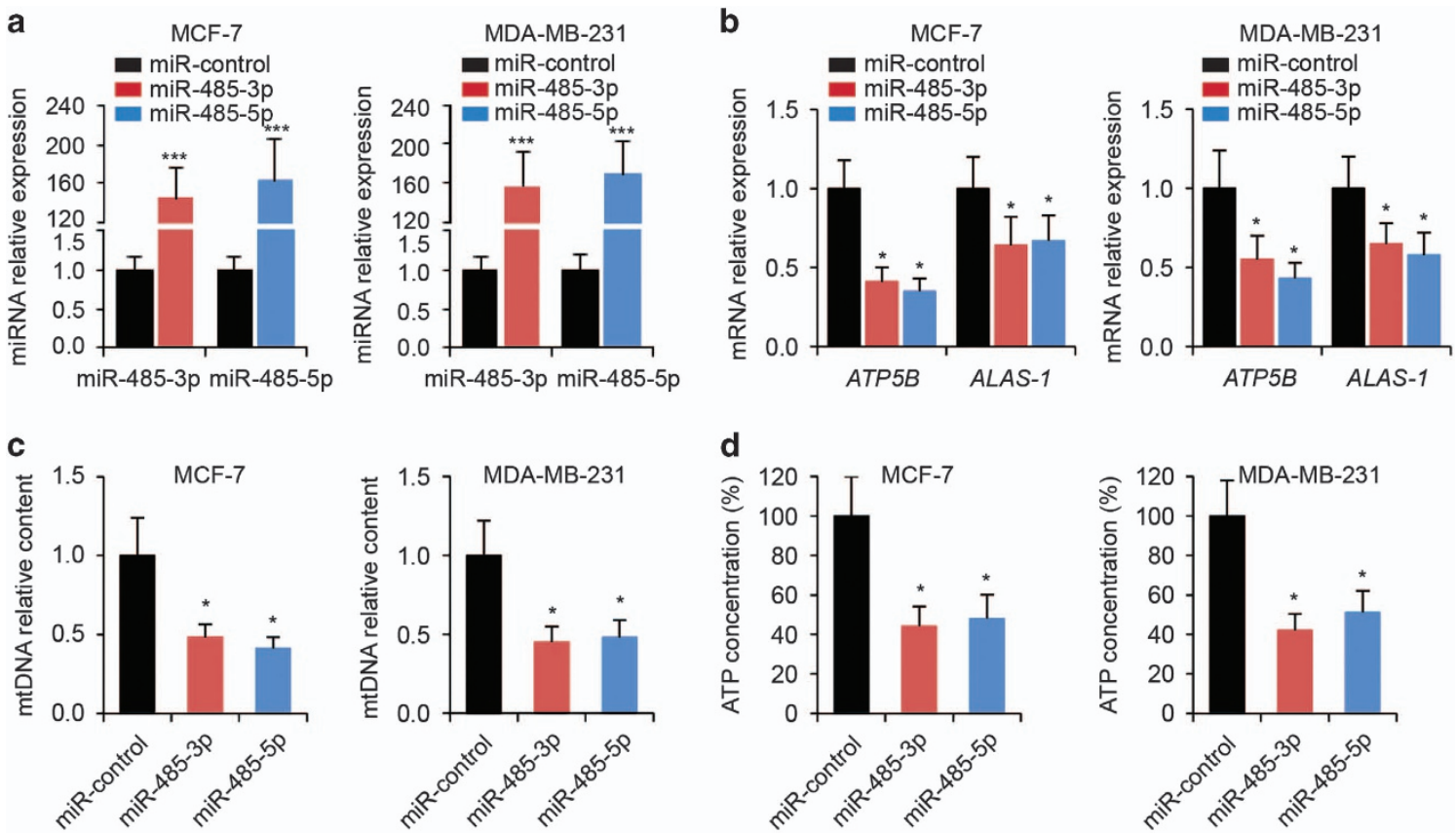

Figure 3 Reduction of mitochondrial respiration in breast cancer cells by miR-485-3p and miR-485-5p. (a) The expression levels of miR-485-3p or miR-485-5p in MCF-7 and MDA-MB-231 cells transfected with miR-control, miR-485-3p or miR-485-5p. ${ }^{* *} \mathrm{P}<0.001$ by $t$-test compared with miR-control-transfected cells. (b) The expression levels of ATP5B and ALAS-1 in MCF-7 and MDA-MB-231 cells transfected with miR-control, miR-485-3p or miR-485-5p. (c) Relative mtDNA content and (d) ATP concentrations in MCF-7 and MDA-MB-231 cells transfected with miR-control, miR-485-3p or miR-485-5p. ${ }^{*} P<0.05$ by one-way ANOVA compared with miR-control-transfected cells

inhibited both migration (Figure 4a) and invasion (Figure 4b) of MCF-7 and MDA-MB-231 breast cancer cells. In addition, we established MDA-MB-231 cells stably expressing miR-control, miR-485-3p and miR-485-5p (Figure 5a), and injected these cells into the tail veins of mice and examined the lung metastasis of the xenograft breast tumors. We determined that both miR-485-3p and miR-485-5p were able to reduce the metastatic area (Figure $5 \mathrm{~b}$ ) and the numbers of nodules in the lung (Figure 5c). To determine whether miR-485-3p and miR-485-5p affect breast cancer cell proliferation, the MTT assay and cell cycle analysis were performed and saw that both miR-485-3p and miR-485-5p suppressed cell proliferation and arrested more cells in the G0/G1 phases (Supplementary Figure 3). Taken together, these data indicated that miR-485-3p and miR-485-5p were involved in regulating mitochondrial respiration, metastasis and proliferation of breast cancer cells.

Restoration of PGC-1a relieves the suppression of mitochondrial respiration, migration and invasion of breast cancer cells mediated by miR-485-3p and miR-485-5p. If miR-485-3p and miR-485-5p suppress mitochondrial respiration, migration and invasion of breast cancer cells through targeting and inhibiting PGC-1a expression, restoration of PGC-1a expression should be able to relieve the suppression. To test this hypothesis, we transfected PGC-1 $a$ into MCF-7 cells and subjected them to miR-485-3p or miR-485-5p transfection. Transfection of PGC-1a evidently restored the protein levels of PGC-1a reduced by miR-485-3p or miR-485-5p transfection (Figure 6a). Significantly, the restoration of PGC-1a expression partially reversed the suppression of gene expression of ATP5B (Figure 6b) and
ALAS-1 (Figure 6c) induced by miR-485-3p or miR-485-5p overexpression. In addition, the restoration of PGC-1a expression also partially relieved the reduction of mtDNA content (Figure 6d) and ATP concentrations (Figure 6e) in MCF-7 cells with miR-485-3p or miR-485-5p overexpression. Furthermore, we found that restoration of PGC-1a expression in MCF-7 cells was also able to reverse the inhibition of cell migration (Figure 7a) and cell invasion (Figure 7b) caused by the overexpression of miR-485-3p and miR-485-5p. These results were consistent with the hypothesis that in breast cancer cells, miR-485-3p and miR-485-5p regulate mitochondrial respiration, cell migration and invasion, at least partially, by inhibiting the expression of PGC-1a.

\section{Discussion}

In this study, we first demonstrated that the expression levels of the mature products of miRNA-485, miRNA-485-3p and miRNA-485-5p were significantly downregulated in breast cancer tissues, and that the expression levels of miRNA-485$3 p$ and miRNA-485-5p were inversely correlated with the potential for metastatic dissemination in breast cancer patients. These results were consistent with recent findings that the expression levels of tumor-suppressing miRNAs were often downregulated in metastatic breast cancer. For example, miR-206 exhibited crucial tumor-suppressor roles in the progression of breast cancer by regulating cyclin-D2 expression. ${ }^{17}$ In addition, miR-720, which acts as a tumor suppressor by inhibiting cell migration and invasion, was found to be downregulated in primary breast carcinoma. ${ }^{18}$ The expression levels of $\mathrm{miR}-485$ itself were also demonstrated by recent studies to be correlated with cancer risk and could 
a
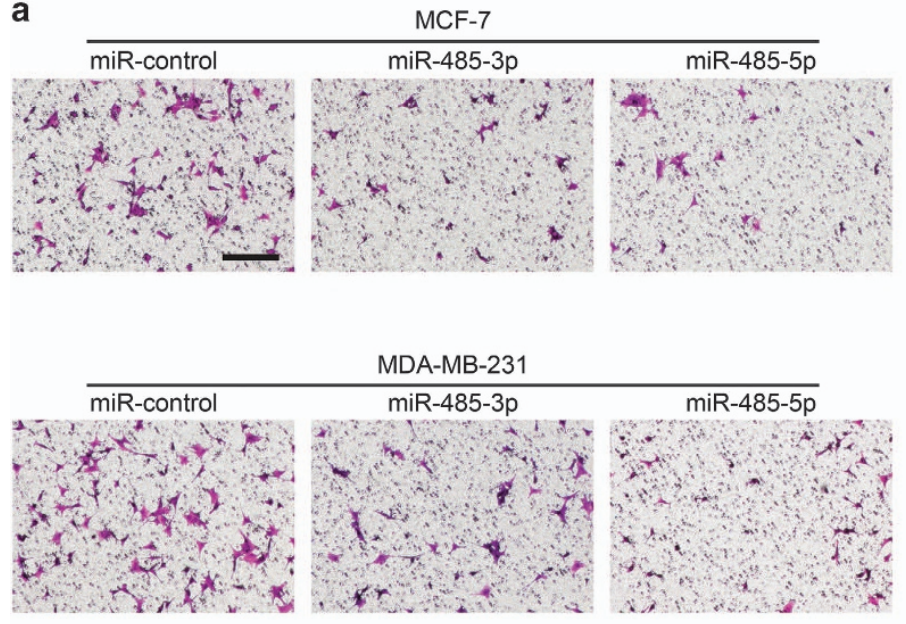

b
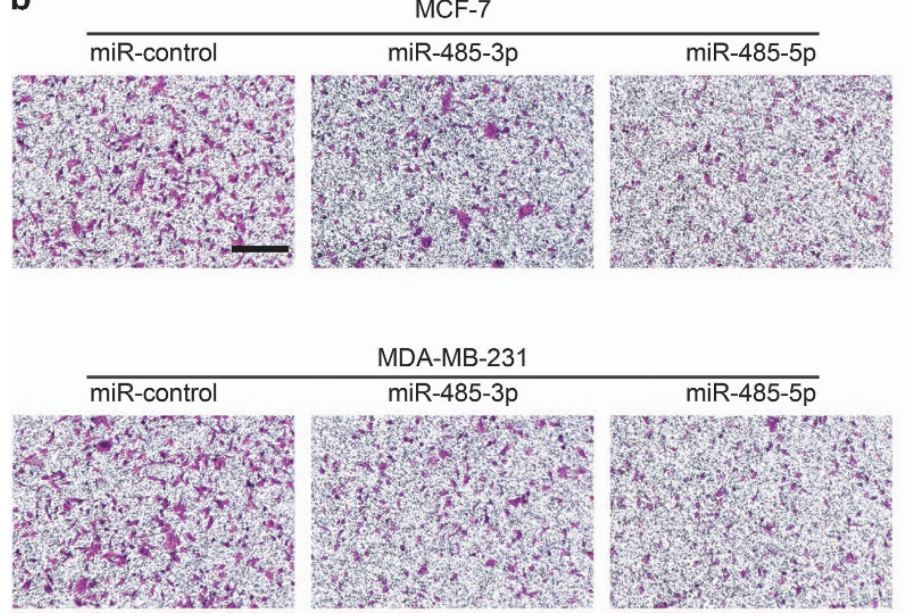
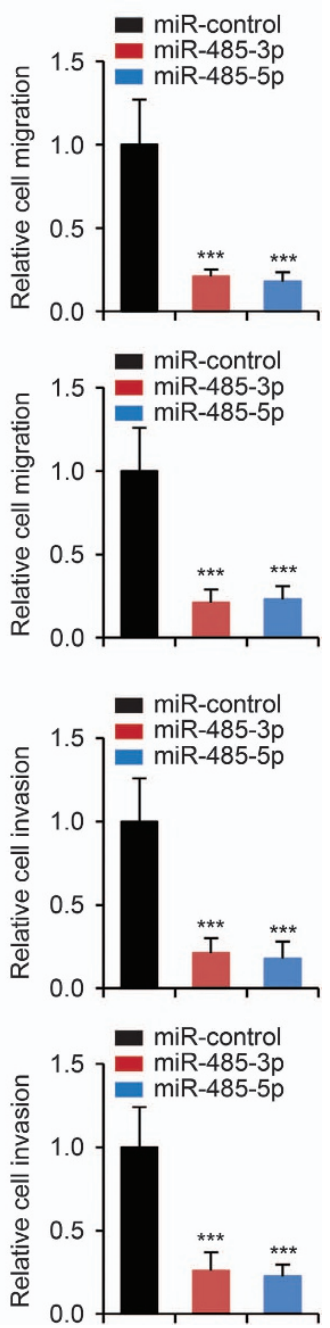

Figure 4 Inhibition of breast cancer cell migration and invasion by miR-485-3p and miR-485-5p. (a) Migration and (b) invasion assays in MCF-7 and MDA-MB-231 cells transfected with miR-control, miR-485-3p or miR-485-5p. Scale bars: $100 \mu \mathrm{m}$. Cell migration and invasion results were normalized by cell proliferation results under the same treatment conditions. ${ }^{* * *} P<0.01$ by one-way ANOVA compared with miR-control-transfected cells

serve as biomarkers for diagnosis of multiple types of cancers. It was reported that the expression of miR-485-5p was reduced in pediatric central nervous system tumors. ${ }^{19}$ In addition, there was a substantial correlation between miR-485 expression and pathological parameters in ovarian cancers, ${ }^{20}$ suggesting that miR-485 could be a reliable biomarker for ovarian cancers. Moreover, miR-485 has also been shown to mediate drug responsiveness in human leukemic lymphoblastic cells and rhabdomyosarcoma cells. ${ }^{21}$

Our study demonstrated that miR-485-3p and miR-485-5p were involved in regulating mitochondrial respiration, cell migration and cell invasion in breast cancer cells by directly targeting and inhibiting the expression of PGC-1a. PGC-1a has recently emerged as an important regulator of cancer metabolism, and as advanced therapeutic strategies are starting to focus on the unique metabolism patterns of cancer cells, PGC-1a has become a potential target for cancer therapy. In a recent study, it was shown that PGC-1a-mediated mitochondrial respiration in cancer cells that was functionally essential for metastasis. Migrating and invading cancer cells relied on PGC-1a to enhance mitochondrial respiration during their metastasis. Suppression of PGC-1a significantly impaired mitochondrial respiration and decreased the metastatic potential of cancer cells. ${ }^{6}$ Consistent with these findings, as PGC-1 $a$ is negatively regulated by miR-485-3p and miR-485-5p, overexpression of miR-485-3p and miR-485-5p significantly reduced mitochondrial respiration and cell migration and invasion in breast cancer cells.

The epigenetic relation between miR-485 and PGC-1a was confirmed by a rescue experiment. We replenished the miR-485-induced downregulation of PGC-1a by ectopic PGC-1a overexpression, and this manipulation partially reversed the phenotypes in mitochondrial respiration and cell migration and invasion induced by miR-485 overexpression. These findings indicated that the effects of miR-485-3p and miR-485-5p on mitochondrial respiration and cell migration and invasion occurred at least partially via a pathway involving PGC-1a. The fact that restoration of PGC-1a could not completely reverse the phenotypes induced by miR-485-3p and miR-485-5p suggested that there could be other signaling 

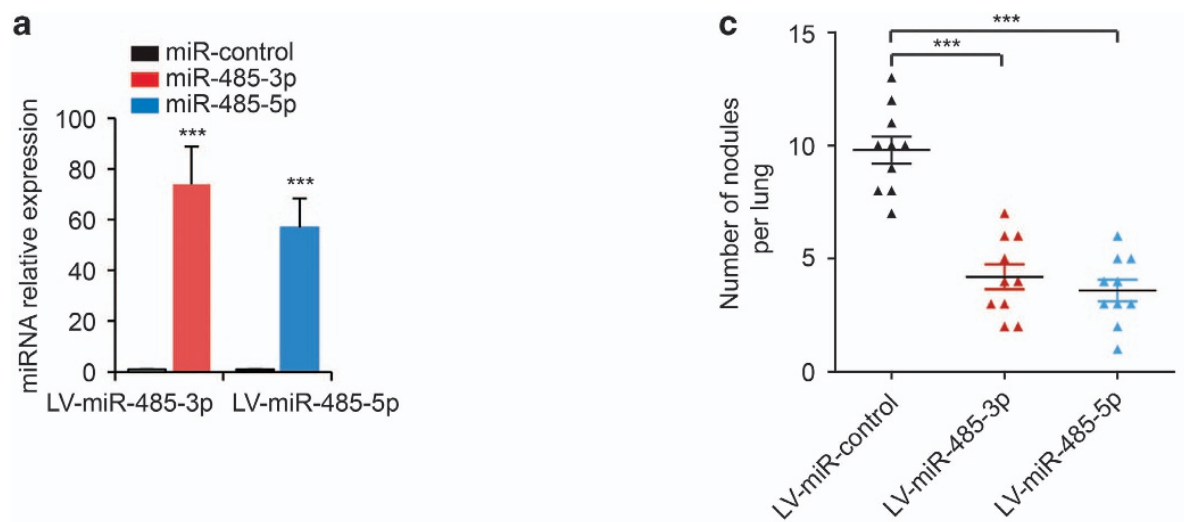

b
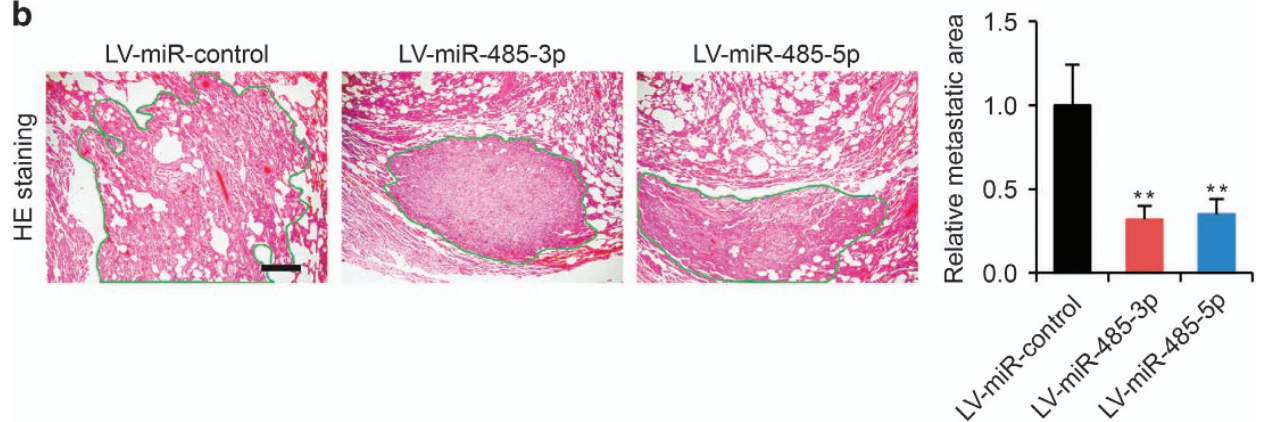

Figure 5 Inhibition of spontaneous lung metastatic capabilities of breast cancer cells by miR-485-3p and miR-485-5p. (a) The expression levels of miR-485-3p or miR-485-5p in MDA-MB-231 cells transduced with LV-miR-control, LV-miR-485-3p or LV-miR-485-5p. (b) Representative photos of H\&E staining of lung tissue sections from mice subjected to tail vein injection of MDA-MB-231 cells stably expressing miR-control (LV-miR-control), miR-485-3p (LV-miR-485-3p) or miR-485-5p (LV-miR-485-5p), and quantification of relative metastatic lung surface area. (c) The quantification of metastatic nodules in lungs from mice subjected to tail vein injection of MDA-MB-231 cells stably expressing miR-control (LV-miR-control), miR-485-3p (LV-miR-485-3p) or miR-485-5p (LV-miR-485-5p). Scale bars: $200 \mu \mathrm{m}$. ${ }^{* \star} P<0.05,{ }^{\star * \star} P<0.001$ by one-way ANOVA compared with the LV-miR control group

pathways downstream of miR-485-3p and miR-485-5p participating in the regulation of mitochondrial respiration and cell migration and invasion in breast cancer. Noticeably, a recent study showed that the miR-485-5p binding site, SNP rs8752, in the HPGD gene was associated with breast cancer risk. ${ }^{22}$

In addition, although miR-485-3p and miR-485-5p appeared to have a suppressive role in mitochondrial respiration and cell migration and invasion, their functions in other aspects of cancer development, such as cell growth and proliferation, are not understood. In this context, it is interesting to note that PGC-1a-mediated enhanced mitochondrial respiration and oxidative phosphorylation did not appear to impact upon glycolytic and anabolic rates, and did not affect cancer cell proliferation or the kinetics of primary tumor growth. ${ }^{6}$ Therefore, the roles of miR-485-3p and miR-485-5p in cancer cell growth and proliferation require further investigation.

\footnotetext{
Materials and Methods

Patients. A total of 30 patients with histologically confirmed breast cancer at Harbin Medical University Cancer Hospital were enrolled in this study from 2011 to 2012. All patients underwent surgical resection without prior radiotherapy or chemotherapy. Primary breast tumors, paired adjacent normal breast tissue (breast tissue $>3 \mathrm{~cm}$ from the tumor) and lymph nodes were collected from each patient. Tissue samples were collected immediately, snap frozen in liquid nitrogen and stored at $-80^{\circ} \mathrm{C}$ for analysis. Informed consent was obtained from all participants. The study was approved by the institutional review board at Harbin Medical University.
}

Cell culture and transfection. Breast cancer cell lines (MCF-7 and MDA-MB-231) and 293 T cells were purchased from the Cell Bank of Type Culture Collection of the Chinese Academy of Sciences, Shanghai, China. The cells were cultured in high-glucose Dulbecco's modified Eagle's medium (DMEM; Gibco, Grand Island, NY, USA) containing $10 \%$ heat-inactivated fetal bovine serum (FBS; Gibco), $100 \mathrm{U} / \mathrm{ml}$ penicillin, $100 \mathrm{U} / \mathrm{ml}$ streptomycin and $2 \mathrm{mmol} / \mathrm{l} \mathrm{L-glutamine,}$ at $37^{\circ} \mathrm{C}$ in a humidified $5 \% \mathrm{CO}_{2}$ atmosphere.

Plasmids and miRNA were transfected in antibiotic-free Opti-MEM medium (Life Technologies, Carlsbad, CA, USA) with lipofectamine 2000 reagent (Life Technologies) according to the manufacturer's instructions. The full-length cDNA of PGC- $1 \alpha$ without the $3^{\prime}$-UTR region was amplified from human genomic DNA and cloned into pcDNA to generate the pcDNA-PGC-1 $\alpha$ constructs. All functional assays were performed $24 \mathrm{~h}$ after transfection.

Western blot analysis. Total proteins of cultured cells were extracted using RIPA buffer $(50 \mathrm{mM}$ Tris $\mathrm{HCl}, 137 \mathrm{mM} \mathrm{NaCl}, 0.5 \% \mathrm{Na}$ deoxycholate, $1 \%$ Triton $\mathrm{X}-100,0.1 \% \mathrm{SDS}$, protease inhibitors) at $4{ }^{\circ} \mathrm{C}$ for $30 \mathrm{~min}$. The cell lysates were centrifuged at $4{ }^{\circ} \mathrm{C}$ for $10 \mathrm{~min}$ at $12000 \mathrm{~g}$ to separate soluble proteins, and the protein concentrations were determined using a bicinchoninic acid protein assay kit (Tiangen Biotech Co., Beijing, China). Proteins were resolved by 10\% SDS-PAGE and transferred onto a nitrocellulose membrane. The membrane was blocked with $5 \%(\mathrm{w} / \mathrm{v})$ non-fat powdered milk in TBS containing $0.1 \%$ Tween for $1 \mathrm{~h}$, washed with TBS/Tween, incubated overnight at $4{ }^{\circ} \mathrm{C}$ with antibodies against PGC- $1 \alpha$ and $\beta$ actin (Santa Cruz Biotechnology, Dallas, TX, USA), and incubated for $2 \mathrm{~h}$ with appropriate secondary antibodies. Signals were visualized by chemiluminescence (Beyotime, Beijing, China).

qRT-PCR. Total RNA was extracted from breast cancer cells and tissues with the TRIzol RNA isolation system (Life Technologies). For miRNA expression, qRT-PCR was performed using TaqMan miRNA assays with specific primer sets (Applied Biosystems, Foster City, CA, USA) according to the manufacturer's protocols. The 

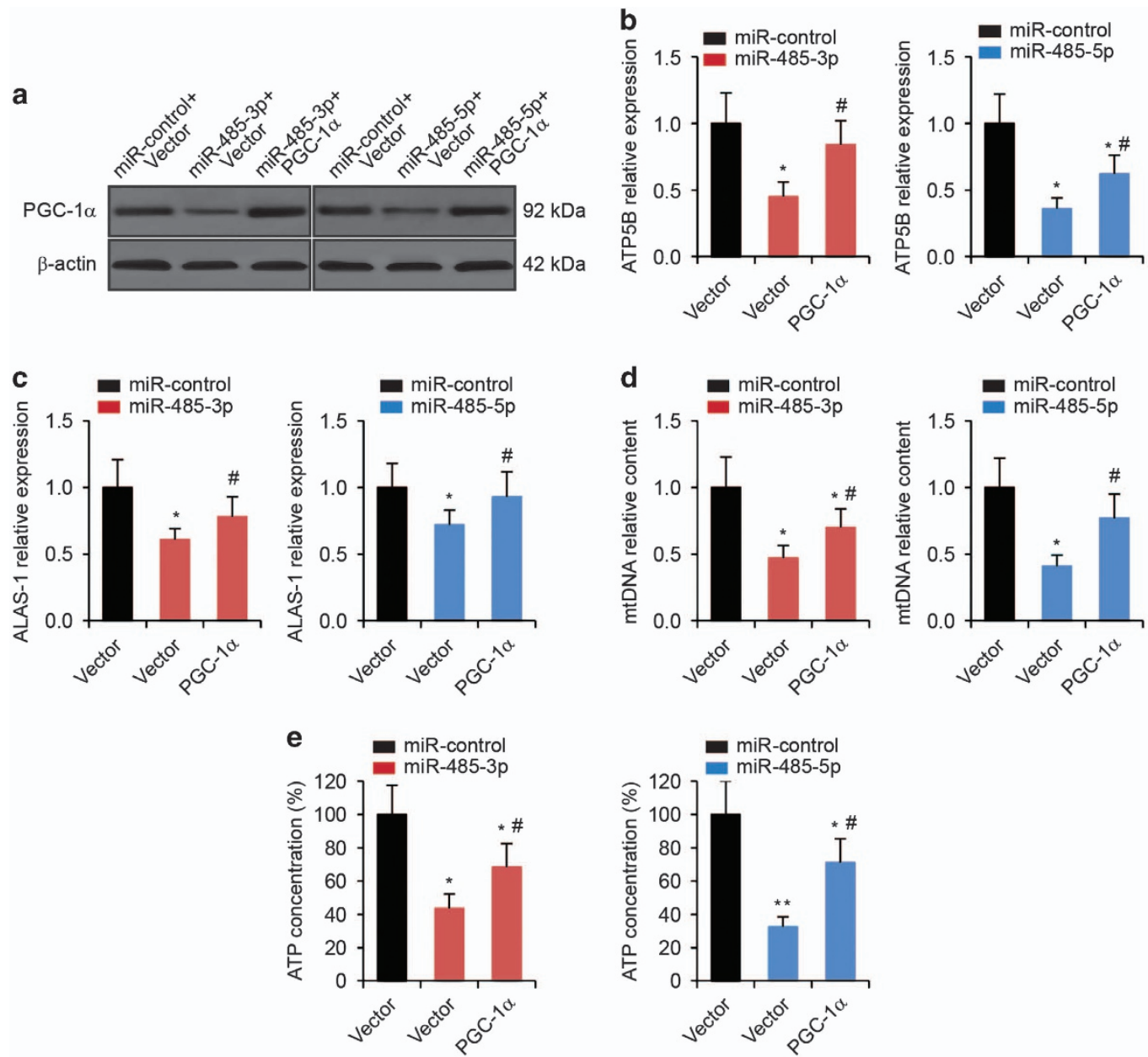

Figure 6 Restoration of PGC-1 $\alpha$ reverses the suppression of mitochondrial respiration induced by miR-485-3p and miR-485-5p. (a) Western blot analysis in MCF-7 cells cotransfected miR-485-3p or miR-485-5p with either empty vector or PGC-1 $\alpha$ plasmid. (b) The expression levels of ATP5B and (c) ALAS-1 in MCF-7 cells as determined by qRTPCR analysis. (d) Relative mtDNA content and (e) ATP concentrations in MCF-7 cells. ${ }^{*} P<0.05,{ }^{* \star} P<0.01$ compared with miR-control-transfected and empty vector-transfected cells by one-way ANOVA. ${ }^{\#} P<0.05$ compared with miRNA-transfected and empty vector-transfected cells by one-way ANOVA

PrimeScript One-step RT-PCR Kit (Takara, Dalian, Liaoning, China) was used for mRNA amplification.

The primers used were as follows: ATP5B: forward primer $5^{\prime}$-TCACCC AGGCTGGTTCAGA-3', reverse primer 5'-AGTGGCCAGGGTAGGCTGAT-3'; ALAS-1: forward primer 5'-GGCAGCACAGATGAATCAGAGAG-3', reverse primer 5'-TTCAGCAACCTCTTTCCTCACGG-3'; PGC-1 $\alpha$ : forward primer 5'-AACAGCA GCAGAGACAAATGCACC-3', reverse primer 5'-TGCAGTTCCAGAGAGTTCCAC ACT-3'; SENP1: forward primer 5'-AAGATTCCCAGACTCCAACTCCCA-3', reverse primer 5'-TGAATGTTCCCGCTCCTGCAAT-3'; TCF7: forward primer 5'-CGTTC CTTCCGATCAGT-3', reverse primer 5'-AGGGCTAGTAGGCAGTTCTGTG-3'; Spred2: forward primer $5^{\prime}$-GAAGGTACCCGGACAGGAAGATGAAGGG-3', reverse primer 5'-GAACTCGAGAGGGAGAAGGGAGGGAAACT-3'; KLF6: forward primer 5'-CTGCCGTCTCTGGAGGAGT-3', reverse primer 5'-TCCACAGATCTTCCTG GCTGTC-3'; Cul3: forward primer 5'-GCCATGGTGATGATTAGAGACA-3', reverse primer $5^{\prime}$-CCGTACCAACTTGATCTCGAAAA-3'; $\beta$-actin: forward primer $5^{\prime}$-GGCT GTATTCCCCTCCATCG-3', reverse primer 5'-CCAGTTGGTAACAATGCCATG-3'.

Luciferase assays. The wild-type (WT) $3^{\prime}$-UTRs of human PGC-1 $\alpha$ and a mutated (MT) $3^{\prime}$-UTR of human PGC- $1 \alpha$ containing mutations in the predicted miR-485 seed regions were amplified and subcloned into the psiCHECK-2 luciferase reporter vector (Promega, Madison, WI, USA). For the luciferase activity assay, 293 T cells were plated into 24-well plates and transfected with psiCHECK-2PGC- $1 \alpha-3^{\prime}$ UTR-WT or psiCHECK-2-PGC- $1 \alpha$-3'UTR-MT, with miR-control, miR-485-3p or miR-485-5p. A Renilla luciferase vector (Promega) was co- transfected to normalize for transfection efficiency. After $48 \mathrm{~h}$ of transfection, firefly and Renilla luciferase activity was determined by the Dual-Luciferase Reporter Assay System (Promega). Relative firefly luciferase activity was measured by normalizing to the Renilla luciferase activity.

Determination of mtDNA content. Total genomic DNA was extracted from breast cancer cells, using the Qiagen DNeasy Kit (Qiagen, Valencia, CA, USA) according to the manufacturer's protocol. The quality of DNA was determined using the Nanodrop spectrophotometer (ThermoFisher Scientific, Rockford, IL, USA). To quantify mtDNA copy numbers in peripheral blood, aRT-PCR was performed using SYBR Green reagents. The amplification of DNA content was obtained using specific primers for a mitochondria encoded gene (ND1). A nuclear encoded gene (HGB) was also quantified and used as control. The amplification of the genes and the level of mtDNA content were determined by qRT-PCR. The primers used were as follows: ND1: forward primer 5'-CCCTAAAACCCGCCACATCT-3', reverse primer 5'-GAGCGATGGTGAGAGCTAAGGT-3'; HGB: forward primer 5'-GTGCACC TGACTCCTGAGGAGA-3', reverse primer 5'-CCTTGATACCAACCTGCCCAG-3'.

Determination of cellular ATP concentrations. Cellular ATP concentrations were measured in breast cancer cells using a bioluminescence assay kit (Promega) according to the manufacturer's instructions. Briefly, the reaction generated signals proportionate to ATP content. The bioluminescence was measured using a microplate multimode reader (Promega). 

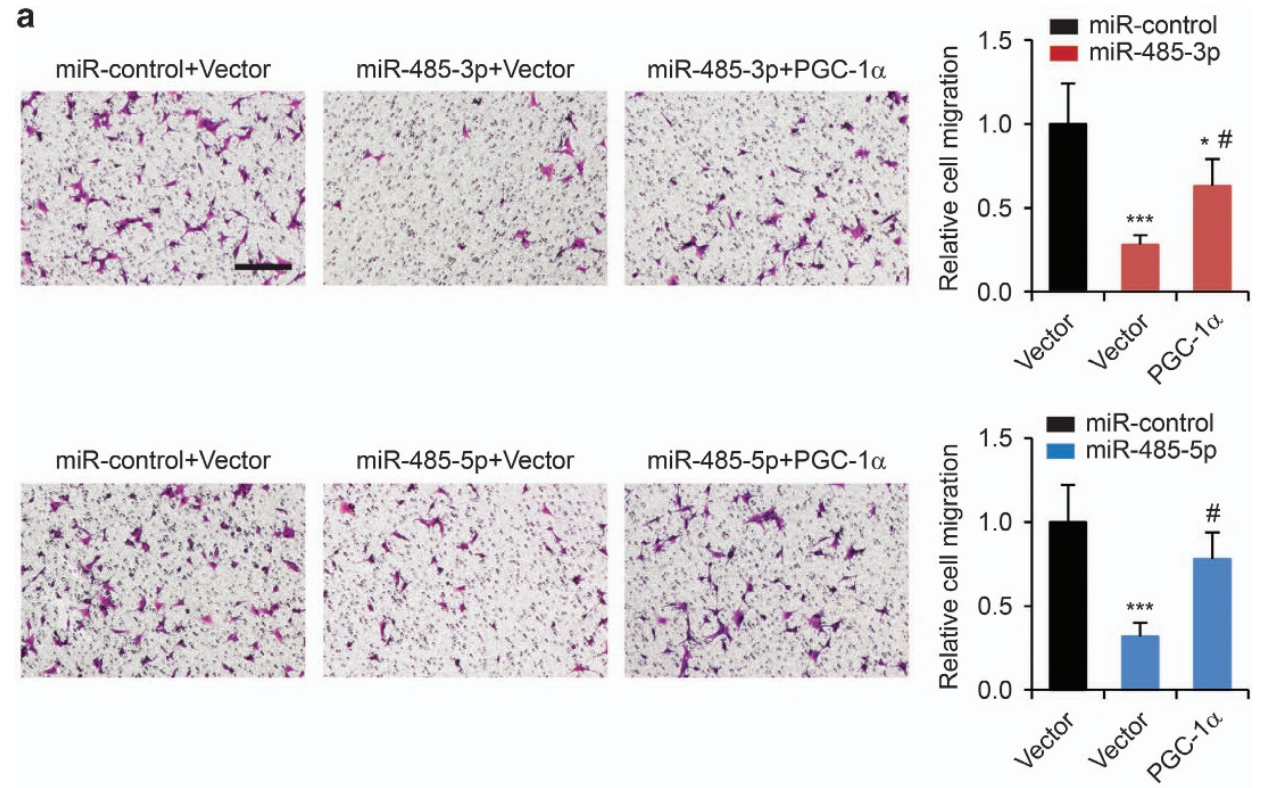

b
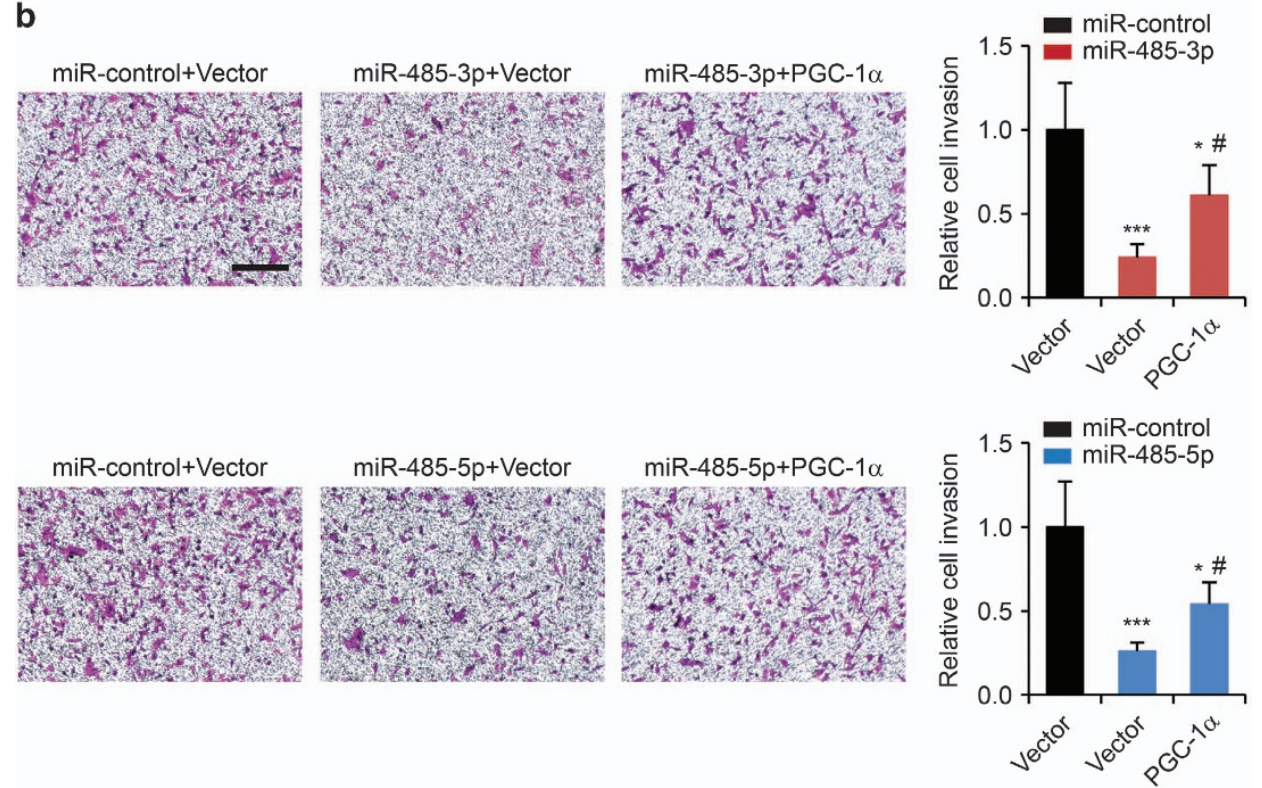

Figure 7 Restoration of PGC-1 $\alpha$ relieves the inhibition of breast cancer cell migration and invasion induced by miR-485-3p and miR-485-5. (a) Migration and (b) invasion assays in MCF-7 cells co-transfected miR-485-3p or miR-485-5p with either empty vector or PGC-1 $\alpha$ plasmid. Scale bars: $100 \mu$ m. Cell migration and invasion results were normalized by cell proliferation results under the same treatment conditions. ${ }^{*} P<0.05,{ }^{* \star *} P<0.001$ compared with miR-control-transfected and empty vector-transfected cells by one-way ANOVA. ${ }^{\#}<0.05$ compared with miRNA-transfected and empty vector-transfected cells by one-way ANOVA

Migration and invasion assays. Migration and invasion assays were performed using 8- $\mu \mathrm{m}$ pore size transwell inserts (BD Biosciences, San Jose, CA, USA). In the migration assay, cells $\left(1 \times 10^{5}\right)$ in $0.2 \mathrm{ml}$ of serum-free medium were added to the upper chambers, and $0.6 \mathrm{ml}$ of DMEM containing $10 \%$ FBS was added to the bottom chambers. Cells were then cultured for $8 \mathrm{~h}$ in a $37^{\circ} \mathrm{C}$ incubator. For the invasion assay, diluted Matrigel (BD Biosciences) was used to coat the insert chambers' membranes. Cells were cultured for $24 \mathrm{~h}$ under the same conditions. Cells that migrated into or invaded the bottom chambers were fixed with paraformaldehyde, stained with crystal violet and quantified in six random fields.

Xenograft model. MDA-MB-231 cells stably expressing miR-control, miR-485-3p or miR-485-5p were established as previously described. ${ }^{23}$ Human miR-485-3p and miR-485-5p were cloned into the lentivirus expression vector
pLenti6.3/v5-DEST to generate pLenti6.3/N5-DEST-miR-485-3p and pLenti6.3/V5DEST-miR-485-5p. MiR-485-3p, miR-485-5p expression constructs, or control vector were packaged with ViraPower Packaging Mix (ThermoFisher Scientific) in the 293FT packaging cell line and concentrated $48 \mathrm{~h}$ after transfection. MDA-MB-231 cells were infected with control, LV-miR-485-3p or LV-miR-485-5p, and selected by blasticidin. The expression of miR-485-3p or miR-485-5p was determined by real-time PCR.

Animal experiments were performed according to the Guide for the Care and Use of Laboratory Animals of Harbin Medical University. The protocol was approved by the Institutional Animal Care and Use Committee of Harbin Medical University. All surgeries were performed under sodium pentobarbital anesthesia, and all efforts were made to minimize suffering. MDA-MB-231 cells $\left(1.5 \times 10^{6}\right)$ were suspended in $200 \mu \mathrm{l}$ of PBS and injected into the tail vein of each BALB/c male nude mouse 
(6-7 weeks old; 10 mice per group). At day 80 after cell injection, all mice were killed after anesthetic overdose, and their lungs were removed and examined. The nodules in the lung were quantified and subjected to hematoxylin and eosin (H\&E) staining.

Statistics. All experiments were performed at least three times. Data were expressed as mean \pm S.D. Statistical significance was analyzed using GraphPad Prism 5.0 (GraphPad Software, Inc., La Jolla, CA, USA). One-way analysis of variance (ANOVA) was performed for serial analysis, whereas two treatment groups were compared by the unpaired Student's t-test. Differences were considered statistically significant when $P$-value was $<0.05$

\section{Conflict of Interest}

The authors declare no conflict of interest.

Acknowledgements. This work was supported by grants from the Natural Science Foundation of Heilongjiang province (H201334); Natural Science Foundation of Heilongjiang province for youth (QC2015114); and the Science and Technology Research Project of the Department of Education of Heilongjiang Province (12531247)

\section{Author contributions}

$\mathrm{CL}$ performed the experiments, collected and analyzed the data, and wrote the manuscript; MX performed the experiments and collected and analyzed the data; SC performed the experiments and analyzed the data; XL collected and analyzed the data; SJ analyzed the data; YR designed the study and wrote the manuscript; and ZL conceived and designed the study, and reviewed and edited the manuscript.

1. Jemal A, Bray F, Center MM, Ferlay J, Ward E, Forman D. Global cancer statistics. $C A$ Cancer J Clin 2011; 61: 69-90.

2. Scully OJ, Bay BH, Yip G, Yu Y. Breast cancer metastasis. Cancer Genomics Proteomics 2012; 9: 311-320

3. Vander Heiden MG, Cantley LC, Thompson CB. Understanding the Warburg effect: the metabolic requirements of cell proliferation. Science 2009; 324: 1029-1033.

4. Warburg O. On the origin of cancer cells. Science 1956; 123: 309-314.

5. Locasale JW, Cantley LC. Metabolic flux and the regulation of mammalian cell growth. Cell Metab 2011; 14: 443-451.

6. LeBleu VS, O'Connell JT, Gonzalez Herrera KN, Wikman H, Pantel K, Haigis MC et al. PGC-1alpha mediates mitochondrial biogenesis and oxidative phosphorylation in cancer cells to promote metastasis. Nat Cell Biol 2014; 16: 1001-1015.

7. Bertoli G, Cava C, Castiglioni I. MicroRNAs: new biomarkers for diagnosis, prognosis, therapy prediction and therapeutic tools for breast cancer. Theranostics 2015; 5: 1122-1143.

8. Winter J, Jung S, Keller S, Gregory RI, Diederichs S. Many roads to maturity: microRNA biogenesis pathways and their regulation. Nat Cell Biol 2009; 11: 228-234.
9. Calin GA, Croce CM. MicroRNA signatures in human cancers. Nat Rev Cancer 2006; 6 : 857-866.

10. Liu YN, Yin J, Barrett B, Sheppard-Tillman H, Li D, Casey OM et al. Loss of androgenregulated microRNA 1 activates SRC and promotes prostate cancer bone metastasis. Mol Cell Biol 2015; 35: 1940-1951.

11. Zhang Q, Tang Q, Qin D, Yu L, Huang R, Lv G et al. Role of microRNA 30a targeting insulin receptor substrate 2 in colorectal tumorigenesis. Mol Cell Biol 2015; 35: 988-1000.

12. Li X, Gong X, Chen J, Zhang J, Sun J, Guo M. miR-340 inhibits glioblastoma cell proliferation by suppressing CDK6, cyclin-D1 and cyclin-D2. Biochem Biophys Res Commun 2015; 460 : 670-677.

13. Labbaye C, Testa U. The emerging role of MIR-146 A in the control of hematopoiesis, immune function and cancer. J Hematol Oncol 2012; 5: 13.

14. Anaya-Ruiz M, Bandala C, Perez-Santos JL. miR-485 acts as a tumor suppressor by inhibiting cell growth and migration in breast carcinoma T47D cells. Asian Pac J Cancer Prev 2013; 14 : 3757-3760

15. Ambros V. microRNAs: tiny regulators with great potential. Cell 2001; 107: 823-826.

16. Shao D, Liu Y, Liu X, Zhu L, Cui Y, Cui A et al. PGC-1 beta-regulated mitochondrial biogenesis and function in myotubes is mediated by NRF-1 and ERR alpha. Mitochondrion 2010; 10: 516-527.

17. Zhou J, Tian Y, Li J, Lu B, Sun M, Zou Y et al. miR-206 is down-regulated in breast cancer and inhibits cell proliferation through the up-regulation of cyclinD2. Biochem Biophys Res Commun 2013; 433: 207-212.

18. Li LZ, Zhang CZ, Liu LL, Yi C, Lu SX, Zhou X et al. miR-720 inhibits tumor invasion and migration in breast cancer by targeting TWIST1. Carcinogenesis 2014; 35: 469-478.

19. Costa FF, Bischof JM, Vanin EF, Lulla RR, Wang M, Sredni ST et al. Identification of microRNAs as potential prognostic markers in ependymoma. PloS One 2011; 6: e25114.

20. Kim TH, Kim YK, Kwon Y, Heo JH, Kang H, Kim G et al. Deregulation of miR-519a, 153, and 485-5p and its clinicopathological relevance in ovarian epithelial tumours. Histopathology 2010; 57 : 734-743.

21. Chen $C F$, He $X$, Arslan AD, Mo YY, Reinhold WC, Pommier $Y$ et al. Novel regulation of nuclear factor-YB by miR-485-3p affects the expression of DNA topoisomerase llalpha and drug responsiveness. Mol Pharmacol 2011; 79: 735-741.

22. He N, Zheng H, Li P, Zhao Y, Zhang W, Song $F$ et al. miR-485-5p binding site SNP rs8752 in HPGD gene is associated with breast cancer risk. PloS One 2014; 9: e102093.

23. Lieber D. Generation of a stable cell line for constitutive miRNA expression. Methods Mol Biol 2013; 1064: 183-200.

published by Nature Publishing Group. This work is licensed under a Creative Commons Attribution 4.0 International License. The images or other third party material in this article are included in the article's Creative Commons license, unless indicated otherwise in the credit line; if the material is not included under the Creative Commons license, users will need to obtain permission from the license holder to reproduce the material. To view a copy of this license, visit http://creativecommons.org/licenses/by/4.0/

Supplementary Information accompanies this paper on Cell Death and Disease website (http://www.nature.com/cddis) 